Niel Khangel S. Reyes, MD

Department of Otorhinolaryngology

Head and Neck Surgery

Victoriano Luna Medical Center

Armed Forces of the Philippines Health Service Command

\section{Post-Operative Bleeding in Tonsillectomy versus Tonsillectomy with Fossa Closure in a Tertiary Military Hospital: A Cohort Study}

\begin{abstract}
Objective: To determine the incidence of post-operative bleeding among patients who underwent tonsillectomy alone versus tonsillectomy with fossa closure at the Victoriano Luna Medical Center from January 2015 to December 2017.
\end{abstract}

\section{Methods:}

\section{Design: Retrospective Cohort Study}

Setting: Tertiary Military Hospital

Patients: Medical records of 83 patients that underwent tonsillectomy under the Department of Otorhinolaryngology - Head and Neck Surgery between January 2015 to December 2017 were retrospectively reviewed for data regarding sex, age, tonsillectomy with or without fossa closure and post-operative bleeding. Cases of tonsillectomy alone versus tonsillectomy with fossa closure were compared (particularly with respect to post-operative bleeding), tabulated and statistically analyzed using risk ratio and t-test.

Results: There were 57 cases of tonsillectomy alone versus 26 cases of tonsillectomy with fossa closure. The incidence of bleeding in all cases of tonsillectomy whether tonsillectomy alone or with fossa closure was $4.8 \%$. The incidence of bleeding was higher in cases of tonsillectomy with fossa closure at $11.5 \%$ (versus $1.8 \%$ in tonsillectomy alone). Post-operative bleeding was 0.1 times more likely to occur in patients who underwent tonsillectomy alone than those who underwent tonsillectomy with fossa closure but there was no statistically significant difference in the risk of post-operative bleeding between the two.

The author declared that this represents original mate that is not being considered for publication or has not been published or accepted for publication elsewhere in full or in part, in print or electronic media; that the manuscript has been read and approved by the author, that the requirements for authorship have been met by the author, and that the author believes that the manuscript represents honest work.

The author signed disclosures that there are no financial or other (including personal) relationships, intellectual passion, political or religious beliefs, and institutional affiliations that might lead to a conflict of interest.

Presented at the Philippine Society of Otolaryngology-Head and Neck Surgery, Analytical Research Contest. October 23, 2018. Roma Salon, The Manila Hotel. Manila.

\section{(c) 96}

Conclusion: Although the incidence of bleeding was higher in cases of tonsillectomy with fossa closure, our results suggest that there is no statistically significant difference in risk for postoperative bleeding between tonsillectomy alone or tonsillectomy with fossa closure.

Keywords: tonsillectomy; postoperative bleeding; tonsil pillars; suturing

Tonsillectomy involves excision of the tonsils, whether bilateral or unilateral, leaving the pillars behind without suturing the anterior and posterior pillars together. It is indicated for tonsillar cancer, severe airway obstruction and a curative measure for recurrent tonsillitis. ${ }^{1}$ 
ORIGINAL ARTICLES

The most common and most serious complication is post-operative bleeding $^{2-4}$ while other possible complications include aspiration, pulmonary edema, atlantoaxial subluxation, injury to the eustachian tube, velopharyngeal insufficiency, nasopharyngeal stenosis, and even psychological trauma depending on the age of the patient. ${ }^{5}$ Common symptoms experienced post-operatively include fever, dehydration, uvular edema, otalgia or odynophagia. ${ }^{5}$

Numerous techniques to improve post-operative outcomes have been sought for decades and one method is tonsillectomy with fossa closure. ${ }^{6}$ However, to the best of our knowledge there are no previous local studies regarding the relationship between post-operative hemorrhage after tonsillectomy alone or tonsillectomy with fossa closure based on our search of the HERDIN database.

Thus, this study sought to determine the incidence of post-operative bleeding among patients who underwent tonsillectomy alone versus tonsillectomy with fossa closure in our institution from January 2015 to December 2017, and whether there was a significant relationship between post-operative bleeding and the surgical procedure.

\section{METHODS}

With Institutional Review Board approval, this retrospective cohort analysis of patients that underwent tonsillectomy from January 2015 to December 2017 at the Victoriano Luna Medical Center of the Armed Forces of the Philippines was conducted. Data regarding age, sex, indication, procedure (tonsillectomy with or without fossa closure) and post-operative bleeding (presence of oozing or active bleeding from the post-operative site that may or may not warrant return to the operating room) were gathered from patient records of the Department of Otorhinolaryngology - Head and Neck Surgery.

Patients were grouped according to age less than 18 years old, 18-40 years old and above 40 years old. Other variables such as the health status of patients, co-morbidities (hypertension, diabetes, blood dyscrasias, history of bleeding), and compliance with medications or pre-existing treatment regimens for other diseases, were excluded from the data gathered.

All data were encoded using Microsoft Excel version 14.6.3 (Microsoft Corp., Redmond, WA, USA). Descriptive statistics were used to summarize the gathered data. A two-sample t-test assuming unequal variances was used to test if there was a significant relationship between surgical technique and risk of post-operative bleeding. The relative risk ratio was computed to estimate the extent of the relationship between surgical technique (tonsillectomy alone versus tonsillectomy with fossa closure) used and the occurrence of post-operative bleeding. The confidence interval was set at $95 \%$.

\section{RESULTS}

Records of a total of 83 patients ( 62 males, 21 females) were included in this study. Their ages ranged from 3 to 54 years old with an average age of 27 years.

Fifteen (18.07\%) patients were under the $<18$ age group while 60 $(72.28 \%)$ were in the $18-40$ age group, and the remaining $8(9.63 \%)$ were in the $>40$ age group.

Indications for tonsillectomy were recurrent tonsillitis in 44 (53.01\%), enlarged (Brodsky Grades III and IV) tonsils in 31 (37.35\%), and obstructive sleep apnea in 8 (9.64\%).

Fifty-seven (68.67\%) underwent tonsillectomy alone and 26 (31.32\%) had tonsillectomy with fossa closure.

There were only four (4) post-operative hemorrhages (4.8\%) overall, 3 among 26 patients who had tonsillectomy with fossa closure and 1 among 57 patients with tonsillectomy alone, with incidence rates of $11.5 \%$ and $1.8 \%$, respectively. While our findings suggest that postoperative bleeding was 0.1 times more likely to occur in patients who underwent tonsillectomy alone than those who underwent tonsillectomy with fossa closure, there was no statistically significant difference in the risk of post-operative bleeding between the two (risk ratio [RR] 6.58; $95 \%$ confidence interval [Cl] 0.72 to $60.26 ; p=.089$ ).

\section{DISCUSSION}

The overall incidence of postoperative tonsillectomy hemorrhage in this study regardless of procedure was $4.8 \%$ which is consistent with the reported rates of $0.28-20 \%$ in other studies, ${ }^{7}$ including another local study with a $5.6 \%$ incidence rate for post-operative bleeding. ${ }^{8}$ The incidence of post-operative bleeding among patients who underwent tonsillectomy alone versus tonsillectomy with fossa closure was 1.8 $\%$ and $11.5 \%$, respectively, which fails to reject the null hypothesis of this study. Our findings contrast with those of foreign studies that suggested there were lesser incidences of post-operative hemorrhage in cases wherein fossa closure was done. ${ }^{7,9,10}$

There are several limitations to this study. In our institution, the main surgeons for tonsillectomies were second year residents assisted by first year residents, under the supervision of third- or fourth year residents. Although surgical competence in tonsillectomy may be achieved in the second year of training, ${ }^{11}$ the uniformity of surgical technique as well as skill of the surgeon were not accounted for by the study design. All three cases of post-operative bleeding in tonsillectomy with fossa closure were brought back to the operating room for evacuation of hematoma and ligation of bleeders while the single case of postoperative bleeding in tonsillectomy alone was managed through application of direct pressure and chemical cauterization using silver nitrate stick only. 


\section{ORIGINAL ARTICLES}

Unfortunately, we did not determine the reasons behind surgeons choosing to perform tonsillectomy with fossa closure versus tonsillectomy alone. If fossa closure was performed for tonsillectomies that were bloodier than usual, or that were deemed to potentially bleed postoperatively, that would be an important intervening variable for consideration and should be explored in future studies.

Confounders including past medical history (including bleeding disorders and anti-coagulant medication intake), family history (of bleeding diathesis), personal and social history (including smoking and ethanol ingestion), co-morbidities (such as hypertension, blood dyscrasias, diabetes, and other chronic illnesses), and laboratory results (including platelet count, prothrombin time, liver function tests) were not included in the data gathered, because we presumed that patients cleared for the procedure were stable and any co-morbidity would have been controlled or accounted for at that point. However, we suggest that such variables be investigated in further studies since they may indirectly affect surgical techniques, as well as postoperative hemorrhage.

In conclusion, although the incidence of bleeding was higher in cases of tonsillectomy with fossa closure, our study found no statistically significant difference in risk for post-operative bleeding between tonsillectomy alone or tonsillectomy with fossa closure.

1. Galindo Torres BP, De Miguel Garcia F, Whyte Orozco J. Tonsillectomy in adults: Analysis of indications and complications. Auris Nasus Larynx. 2018 Jun; 45(3); 517-521. DOI: 10.1016/j. anl.2017.08.012; PMID: 28927847.

2. Myssiorek D, Alvi A. Post-tonsillectomy hemorrhage: An assessment of risk factors. Int J Pediatr Otolaryngol. 1996 Sep; 37(1): 35-43. PMID: 8884405.

3. Walker P, Gilles D. Post-tonsillectomy hemorrhage rates: Are they techniquedependent? Otolaryngol Head Neck Surg. 2007 Apr; 136(4 Suppl: S27-S31. DOI: 10.1016/j. otohns.2006.10.022; PMID: 17398338.

4. Windfuhr JP. Serious complications following tonsillectomy: how frequent are they really? ORL J Otorhinolarygol Relat Spec. 2013;75(3): 166-173. DOI: 10.1159/000342317; PMID: 23978803.

5. Gallagher TQ, Wilcox L, McGuire E, Derkay CS. Analyzing factors associated with major complications after adenotonsillectomy in 4776 patients: comparing three tonsillectomy techniques. Otolaryngol Head Neck Surg. 2010 Jun; 142(6): 886-892. DOI: 10.1016/j. otohns.2010.02.019; PMID: 20493363.

6. Matt BH, Krol BJ, Ding Y, Juliar BE. Effect of tonsillar fossa closure on postoperative pain and bleeding risk after tonsillectomy. Int J Pediatr Otolaryngol. 2012 Dec; 76(12): 1799-1805. DOI: 10.1016/j.jporl.2012.09.004; PMID: 23021465

7. Elkholy TA. Modified Surgical Technique with Pillars Repair in Reducing Post Tonsillectomy Haemorrhage. Int Inv J Med Sci. 2016 Jun 3(6): 108-114.

8. Moral SD, Barlin AKC, Acuin JM. Clinical profile of post-tonsillectomy bleeding; A 30-month institutional review. Philipp J Otolaryngol Head Neck Surg. 2010 Jul-Dec 25(2):14-17.

9. Senska G, Schröder H, Pütter C, Dost P. Significantly Reducing Post-Tonsillectomy Haemorrhage Requiring Surgery by Suturing the Faucial Pillars: A Retrospective Analysis. PLoS One. 2012; 7(10): e47874. DOI: 10.1371/journal.pone.0047874; PMID: 23118902; PMCID: PMC3485309.

10. Wulu JA, Chua M, Levi JR. Does suturing tonsil pillars post-tonsillectomy reduce postoperative hemorrhage: A literature review. Int J Pediatric Otorhinolaryngology. 2019 Feb; 117:204-209. DOI: 10.1016/j.ijporl.2018.12.003; PMID: 30611028

11. Ahmed A1, Ishman SL, Laeeq K, Bhatti NI. Assessment of improvement of trainee surgical skills in the operating room for tonsillectomy. Laryngoscope. 2013 Jul;123(7):1639-44. DOI: 10.1002/ lary.24023. PMID: 23483535. 